\title{
Emittance scans for CMS luminosity calibration in Run 2
}

\section{O. Karacheban* on behalf of the CMS Collaboration CERN, Geneva, Switzerland}

E-mail: olena.karacheban@cern.ch

\section{Y.C. Çekmecelioğlu}

Boğaziçi University, Istanbul, Turkey

E-mail: yusuf.can.cekmecelioglu@cern.ch

\begin{abstract}
The absolute luminosity calibration for LHC experiments is derived from dedicated beam separation scans, known as van der Meer (vdM) scans. However, vdM scans are performed with special beam optics, wide beams, and fewer, well-separated bunches to reduce potential systematic effects, and only once per year per collision mode. In order to use the calibration obtained from a vdM scan under physics data-taking conditions with more bunches and significantly higher instantaneous luminosity, an additional measurement of the stability and linearity of the luminometers is required. Potential nonlinear effects are important especially during Run 2 (2015 2018), where pileup during physics data taking reached up to about 50 and above. Short vdM-type ("emittance") scans were thus performed regularly in CMS since 2017 in the $\mathrm{x}$ and y planes in 7 -9 beam separation steps at the beginning and end of each fill. They allowed for powerful performance diagnostics of the luminosity subdetectors in CMS throughout the year. In addition, for the subdetectors that publish luminosity measurements online (BCM1F, HF, and PLT) and read out at $40 \mathrm{MHz}$, emittance scans allow studying effects on a per bunch crossing level, correcting for beam-beam effects per bunch, and separating effects due to sequential bunches ("bunch trains"), as well as monitoring beam evolution during the fill. Analysis techniques and the great potential of emittance scans in Run 3 are illustrated.
\end{abstract}

The European Physical Society Conference on High Energy Physics (EPS-HEP)

10-17 July 2019

Ghent, Belgium

${ }^{*}$ Speaker. 


\section{CMS online luminometers}

To insure reliable uninterrupted luminosity measurement in LHC Run 2, the CMS experiment was equipped with multiple online luminometers. The Beam Radiation Instrumentation and Luminosity (BRIL) project is responsible for the design, production, commissioning, and operation of dedicated luminosity detectors: the Pixel Luminosity Telescope (PLT) [1, 2] and the Fast Beam Condition Monitor (BCM1F) [2]. Polycrystalline diamond (pCVD) sensors and silicon (Si) sensors are used in BCM1F. In addition independent readout of the Forward Hadron (HF) calorimeter was used for luminosity measurement [3].

The number of hits per bunch crossing follows a Poisson distribution. The probability of a certain number of hits for BCM1F (triple coincidences for PLT or towers with signal above the threshold for HF) is described by $p(n)$. The probability of having no hits, triple coincidences or towers with signal is described by $p(0)$. Since the occupancy per channel is low, the "zero counting" method is used to determine the mean value of the number of hits per orbit per bunch crossing, $\mu$, for luminosity calculation as:

$$
p(n)=\frac{\mu e^{-\mu}}{n !}, \quad \mu=-\log [p(0)]=-\log [1-p(\neq 0)] .
$$

The above described HF occupancy method is hereafter referred to as HFOC. HF is also providing luminosity measurement based on the sum of the transverse energy, hereafter referred to as HFET.

\section{CMS luminosity calibration and emittance scans}

Absolute luminosity calibration is performed using van der Meer (vdM) scans [4], special beam separation scans in the $\mathrm{X}$ and $\mathrm{Y}$ planes under specific beam conditions. In principle calibration could be also performed using a physics process, but this would require knowing the cross section in proton-proton collisions to a greater than 1\% precision. The 2017 and $2018 \mathrm{VdM}$ scan programs and full analyses are described in detail in [4,5]. As a vdM program is carried out only once per year per collision mode, an additional handle on the stability of the luminometers calibration is required. From 2017, the so-called "emittance scans" were performed at the beginning of almost every LHC fill [3], hereafter referred to as "early" emittance scans. In the 10-12 hours long fills, which became typical during the LHC operation in 2018, the luminosity dropped by almost a factor of two towards the end of the fill. Therefore, emittance scans in the long fills are also performed at the end of the fill - hereafter referred to as "late" emittance scans - allowing to check the linearity of the detectors. In 2018, emittance scans were done in 9 separation steps, collecting data for $10 \mathrm{~s}$ at each step.

Fig. 1 (left) illustrates $\mu$ values for the HFOC during the emittance scan in X and Y directions in fill 6592. The smaller dips before the emittance scan correspond to beam optimizations, which are performed to find the position where beams collide head-on. Such optimizations became a part of the standard procedure to improve the quality of the emittance scans. A single Gaussian function is used to fit the normalized $\mu$ values as a function of the beam separation in emittance scans, e.g. as shown in Fig. 1 (middle, right). The peak rate in $\mathrm{X}\left(R_{\max , \mathrm{X}}\right)$ and $\mathrm{Y}\left(R_{\max , \mathrm{Y}}\right)$ scans, the peak position, and the effective beam overlap in $\mathrm{X}\left(\Sigma_{X}\right)$ and $\mathrm{Y}\left(\Sigma_{Y}\right)$ are obtained from the fit and used to calculate the visible cross section $\left(\sigma_{\mathrm{vis}}\right)$ for each detector: 

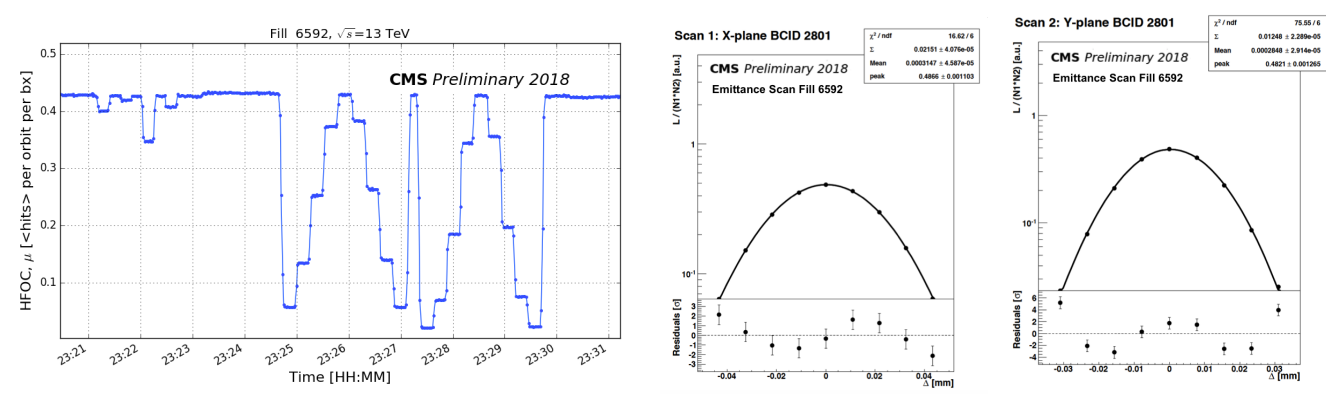

Figure 1: Rate observed by HFOC during an emittance scan in $X$ and $Y$ directions in 9 beam separation steps each (left). Fit of the normalized rate in X plane (middle) and in Y plane (right) to a Gaussian function are shown with the solid line. Dots correspond to averaged and bunch current normalized $\mu$ values per beam separation step.

$$
\sigma_{\mathrm{vis}}=\pi \Sigma_{X} \Sigma_{Y}\left(R_{\max , \mathrm{X}}+R_{\max , \mathrm{Y}}\right)
$$

$\sigma_{\mathrm{vis}}$ is a quantity characterising the detector and stays constant for stable detector configuration. Monitoring of the $\sigma_{\mathrm{vis}}$ during the year allows to indicate periods where detector performance changed and derive the relevant efficiency corrections. Corrections to $\sigma_{\mathrm{vis}}$ to compensate for beambeam effects should be taken into account: deflection of two proton beams results into orbit shift and optical beam distortion on the proton bunch densities (dynamic- $\beta$ effect) [6].

\section{Measurement of $\sigma_{\mathrm{vis}}$ for stability and nonlinearity monitoring}

Fig. 2 shows $\sigma_{\text {vis }}$ for the PLT (left) and BCM1F (right) detectors for 2018 with beam-beam corrections applied ${ }^{1}$. Decreasing $\sigma_{\text {vis }}$ values delineate a reduced detector efficiency which was mitigated by either an increase of the operational high voltage or optimizing detector settings to compensate for the radiation damage. The efficiency corrections for time periods with changing detector performance are derived from the ratio of the $\sigma_{\mathrm{vis}}$ measured in the emittance scan to the reference $\sigma_{\mathrm{vis}}$ that was measured in the vdM scan and applied to calculate the luminosity during complete data-taking period.
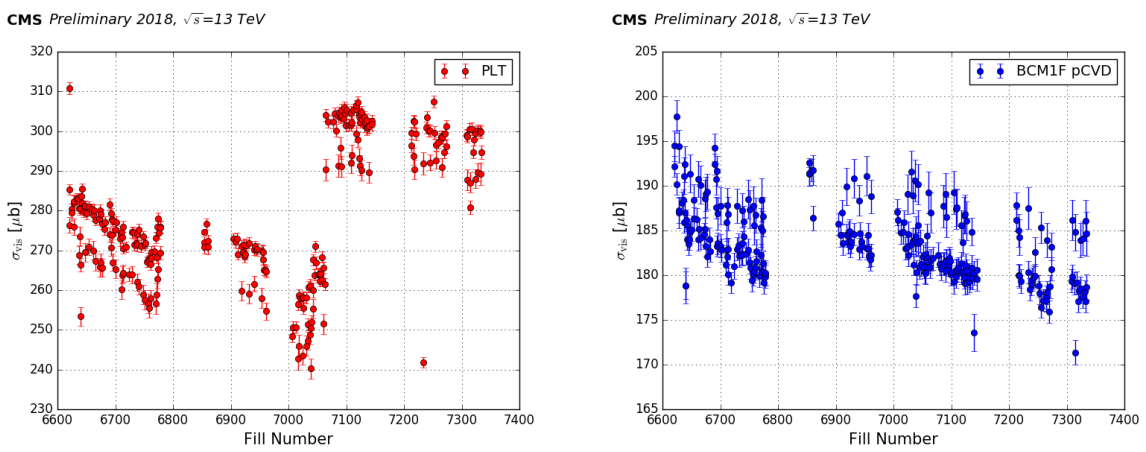

Figure 2: The measured $\sigma_{\mathrm{vis}}$ as a function of fill number for PLT (left) and BCM1F (right) during 2018.

One can distinguish two subgroups in $\sigma_{\mathrm{vis}}$ of PLT and BCM1F pCVD (Fig. 2). The less populated band of points with larger statistical uncertainty correspond to late emittance scans,

\footnotetext{
${ }^{1}$ Calculations are based on using single-particle approach and Gaussian beam approximation [6]. Work is ongoing to derive corrections based on the multi-particle approach and take into account emittance change during the fill.
} 
given they are not performed in every fill. The event count is higher in early emittance scans: band of points with smaller statistical uncertainty. It is worth mentioning that PLT measures lower $\sigma_{\text {vis }}$ in the late scans and BCM1F pCVD measures higher $\sigma_{\mathrm{vis}}$ in the late scans, pointing to the presence of opposite sign nonlinearity in these two detectors.

In the past, it was only possible to measure relative nonlinearity based on the ratios of luminosity reported by different detectors. Employing emittance scans the nonlinearity can be determined in every long fill using the measured $\sigma_{\text {vis }}$ values for each bunch in every early and late emittance scan that cover a wide range of Single Bunch Instantaneous Luminosity (SBIL). In Fig. 3, $\sigma_{\mathrm{vis}}$ is shown for leading (blue) and train (red) bunches as a function of SBIL with linear fits for PLT and BCM1F pCVD. As stated earlier, PLT and BCM1F pCVD have nonlinearity of opposite sign. Averaging through several fills well separated in time, PLT and BCM1F pCVD nonlinearities measured in 2018 from emittance scans are $0.94 \pm 0.08$ (stat) and $-0.67 \pm 0.11$ (stat) \%, respectively, for train bunches and per unit of SBIL. HFOC, HFET and BCM1F Si show almost linear behavior with slightly positive nonlinearity: $0.20 \pm 0.07$ (stat), $0.27 \pm 0.08$ (stat), $0.14 \pm 0.12$ (stat) \%, respectively, per unit of SBIL.

As shown in Fig. 4, the two-dimensional distribution of the SBIL- $\sigma_{\text {vis }}$ plane is quite wide and for detectors with low nonlinearity, emittance scans method reaches its current limit on precision. The $\sigma_{\text {vis }}$ distributions in early and late scans are different for all detectors: they are less uniform at the end of the fill (range of low SBIL on the plots), indicating more spread in bunch parameters, such as bunch shape, emittance, bunch current, etc., and introducing some complications to the nonlinearity analysis.
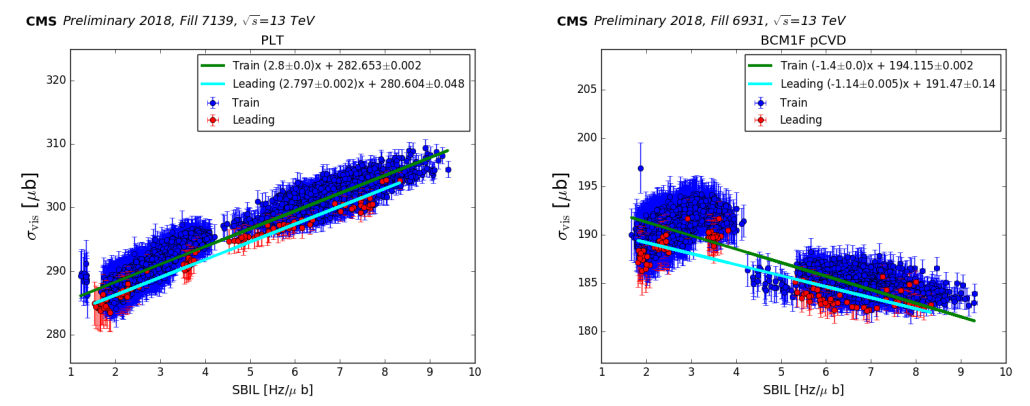

Figure 3: The measured $\sigma_{\text {vis }}$ vs. SBIL for PLT (left) and BCM1F pCVD (right).
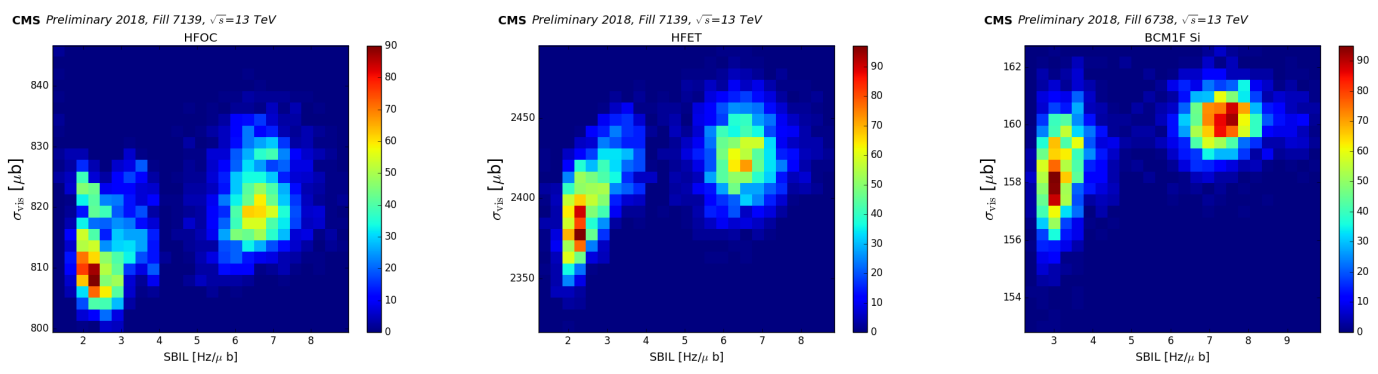

Figure 4: $\sigma_{\text {vis }}$ vs. SBIL for HFOC (left), HFET (middle) and BCM1F Si (right) for train bunches.

To better understand the evolution of the bunches in the bunch trains in the long fills we use the measured beam overlap width for emittance calculation. Comparing emittance measurement in early and late scans, we observe differences in the evolution of the bunch depending on its 
position in the bunch train. As shown in Fig.5, the evolution of the first 10-12 bunches in the train is different from the other bunches, especially noticeable in X. Understanding the measured emittance evolution is also of high importance for benchmarking the simulation code (TRAIN) for the beam evolution prediction [7] and improving the luminosity model [8].
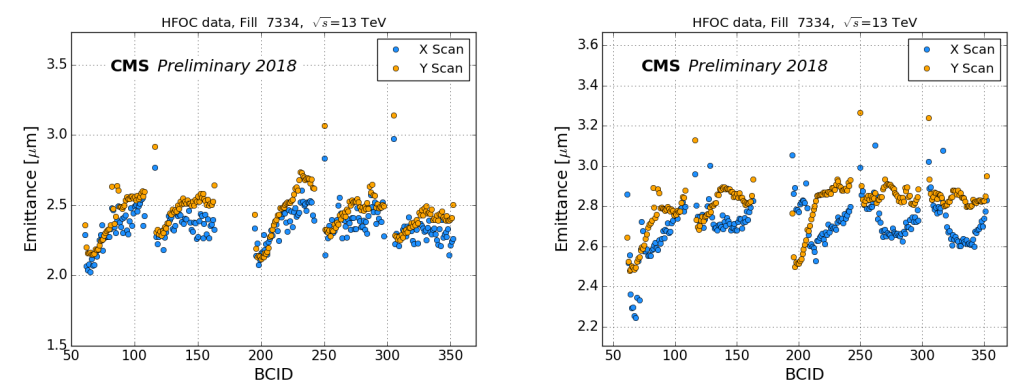

Figure 5: Emittance measured per bunch crossing in early (left) and late (right) emittance scans.

\section{Summary}

CMS emittance scans are shown to be powerful tool for luminosity calibration stability and nonlinearity monitoring. As the instantaneous luminosity will further increase in future LHC runs, precise measurement of nonlinearity will be essential. It is already one of the leading luminosity uncertainties. Analysis of emittance evolution per bunch crossing is of high priority. In particular, it is used for comparison to emittance values measured by other LHC instruments and for improving the LHC simulations and luminosity model for the beam evolution, and hence the overall luminosity prediction.

\section{References}

[1] M. Barbero et al., Design and test of the CMS pixel readout chip, Nucl. Instrum. Methods Phys. Res., A 517 (2004) 349, DOI: 10.1016/j.nima.2003.09.043.

[2] M. Guthoff on behalf of the CMS, Instrumentation for beam radiation and luminosity measurement in the CMS experiment using novel detector technologies, Nucl. Instrum. Methods Phys. Res., Volume 845 (2017) 565-569, DOI: 10.1016/j.nima.2016.06.028.

[3] O. Karacheban, P. Tsrunchev on behalf of CMS, Emittance scans for CMS luminosity calibration, EPJ Web of Conferences 201 (2019) 04001, DOI: 10.1051/epj-conf/201920104001.

[4] CMS Collaboration, CMS Luminosity Measurement for the 2017 data-taking period, CMS-PAS-LUM-17-004, https://cds.cern.ch/record/2621960

[5] CMS Collaboration, CMS luminosity measurement for the 2018 data-taking period at $\sqrt{s}=13 \mathrm{TeV}$, CMS-PAS-LUM-18-002, https://cds.cern.ch/record/2676164

[6] A. Babaev for CMS Collaboration, Beam-dynamic effects at the CMS BRIL van der Meer scans, JINST 13 (2018) C0302 and DOI: 10.1088/1748-0221/13/03/C0302

[7] M. Hostettler et al., Luminosity scans for beam diagnostics, Phys. Rev. Accel. Beams 21, (2018) 102801, DOI: 10.1103/PhysRevAccelBeams.21.102801

[8] F. Antoniou et al., Building a luminosity model for the LHC and HL-LHC, IPAC2015, TUPTY020 (2015) 2042 DOI:10.18429/JACoW-IPAC2015-TUPTY020 